\title{
High-resolution lidar experiment for the Thirty Meter Telescope
}

\author{
Thomas Pfrommer ${ }^{1, \text { a }}$, Paul Hickson ${ }^{1}$, and Chiao-Yao She ${ }^{2}$ \\ 1 Department of Physics and Astronomy, University of British Columbia, Canada \\ 2 Department of Physics, Colorado State University, USA
}

\begin{abstract}
Adaptive optics (AO) systems of extremely-large optical telescopes (ELTs) will employ laser guide stars (LGS) to achieve wide sky coverage. In these systems the mesospheric sodium layer at $90 \mathrm{~km}$ altitude is excited by means of laser-induced fluorescence of the Na I D2 - resonance hyperfine transmission. The finite thickness of the sodium layer and temporal variations in its density structure produce elongation, internal structure, and range variations in the LGS. This degrades the performance of the AO system due to degeneracy between effects of atmospheric and sodium layer variations. In order quantify this and assess the impact on future extremely large telescopes such as the Thirty-Meter Telescope, we have developed a lidar system for the 6-m Large Zenith Telescope. This system provides more than an order-of-magnitude improvement in signal-tonoise ratio and spatio-temporal resolution compared to previous work. Initial observations have revealed new details of the structure and dynamics of the sodium region. These include multiple transitory layers that vary in density and altitude on timescales ranging from minutes to hours, large-scale instabilities, turbulent vortices and coherent short period gravity wave oscillations. The mean sodium altitude exhibits temporal variations having a near-Kolmogorov power spectrum extending over four decades of frequency. The impact of these variations will be significant for all ELTs employing continuous-wave laser beacons.
\end{abstract}

\section{Introduction}

The Sodium layer extends in the atmospheric upper Mesosphere and lower Thermosphere (MLT) in the region from $75 \mathrm{~km}$ to $110 \mathrm{~km}$. It is created by neutral atoms that ablate from meteoric dust in the sub- $\mu \mathrm{g}$ mass range Ceplecha et al. (1998).

Due to its relatively large abundance compared to other metals in the MLT, the relatively high mean altitude of the MLT $(\tilde{9} 2 \mathrm{~km})$, its large fluorescence cross section and resonance transition in the optical wavelength range at $589 \mathrm{~nm}$, Sodium is used to create artificial stars by adaptive optics (AO) systems. These "laser guide stars" (LGS) are cylinders of illuminated sodium atoms.

With the sodium layer's extended height, most points in the entrance pupil of the telescope (normally the primary mirror) will see the LGS spot elongated because of horizontal separation between the laser launch telescope and the observation point. Especially for next generation extremely large telescopes (ELT) this issue becomes severe and needs special attention, which will be further addressed in sect. 2.2.

In addition, a changing mean height of the sodium causes the images of the laser illuminated cylinders in the layer to defocus. The AO system cannot distinguish between defocus caused by the atmosphere and defocus caused by variations in the sodium mean height, making it impossible to keep the science target in focus. Natural guide stars can give additional input and mitigate this problem, it is however very important to know on which timescales the sodium mean height changes.

In a first approach, Davis et al. (2006) investigated the mean height variations in the Sodium layer from existing lidar measurements that have integrated profiles over 2 minutes. They find that the power spectrum of the mean height variations is well represented by a power law, at least at frequencies below $10 \mathrm{mHz}$. Herriot et al. (2006) estimate this power spectrum, if it continues to high frequencies, will contribute significant variance to the residual wavefront error of the TMT Narrow-Field Infrared Adaptive Optics System (NFIRAOS). The validity of extrapolating the low-frequency power law to frequencies as high as $1 \mathrm{kHz}$ is highly uncertain so is essential to determine the temporal behaviour of

\footnotetext{
${ }^{a}$ e-mail: pfrommer@phas.ubc.ca
} the original work is properly cited. 
the sodium density profile at higher frequencies, close to those of the atmospheric focus fluctuations. This is the primary aim of the LZT Lidar Project (Pfrommer et al. (2008)), carried out at the UBC Large Zenith Telescope near Vancouver $\left(49.3^{\circ} \mathrm{N}, 122.6^{\circ} \mathrm{W}\right)$ with its $6 \mathrm{~m}$ primary mirror (Hickson et al. (2007)). This large area ensures the collection of enough photons to get reasonable signal to noise ratio at much shorter time scales than previously reported from other lidar experiments.

\section{The Sodium Layer}

The MLT is a region in the sky, where meteoric ablated metals like $\mathrm{Fe}, \mathrm{Ca}, \mathrm{K}$ and $\mathrm{Na}$ exist in relatively high abundance. Above this layer $\gtrsim 110 \mathrm{~km}$, these metals exist in ionized form, recombining with electrons from the Ionosphere and hence becoming neutral and visible to the lidar. The atoms then migrate downward through the layer of interest, get affected by the predominant weather dynamics and at the lower end $\lesssim 75 \mathrm{~km}$ the density of oxygen is large enough to form molecules and hence removes neutral Sodium from the atmosphere (Plane (2003)).

With temperatures around $180 \mathrm{~K}$, the MLT is the coldest region in the earths atmosphere, shows strong mean zonal winds of up to $50 \mathrm{~m} / \mathrm{s}$, strong wind shear throughout the layer and diurnal and severe seasonal changes (Yuan et al. (2008)). In addition, current studies show evidence that this region is the shore line of gravity waves, meaning, they overturn and dissipate their energy into the surroundings (Hecht et al. (2004), Xu et al. (2006)).

\subsection{Turbulence in the Sodium Layer}

The environmental facts, shown in the last paragraph show that the region that astronomers have chosen to use for LGS systems (because of mentioned advantages in sect. 1) is a complex and highly dynamic region of the atmosphere. Fig. 1 shows the Sodium layer, color coded for the photon return flux, which is apart from a small $h^{2}$-factor (with $h$ being the altitude from the layer to our zenith pointing telescope) proportional to the Sodium density.

Even though only about 1.5 hours of data is shown, many interesting features are visible in this data set. It shows nicely overturning as we have two maxima (individual Sodium clouds on top of a smoother background Sodium distribution). This overturning of gravity waves can be interpreted like the ocean analogy with breaking waves on shore lines. Fig. 1 also shows small scale instabilities with an onset of Kelvin-Helmholtz instabilities, zoomed out in the lower left window and it also shows a turbulent roll extended to about $1 \mathrm{~km}$ in height in the lower right window. It also shows the large range that Sodium layer densities can vary over the course of less than a minute or even seconds, which is of great interest to adaptive optics.

The upper sodium cloud shows photon returns that are about a factor of 3 above the sodium background. This puts this cloud into the region of the so-called sporadic sodium layers (SSL), which are defined as suddenly occurring thin clouds extended to a few $\mathrm{km}$ in height and at least a factor of 2 higher density then the background (Clemesha (1995)).

Often these individual clouds appear over periods of 10 s of seconds, persist for several hours and drift downwards. If their source are gravity waves, then the downward motion is expected as gravity waves have wave phase front velocities whose z-component is directed downwards. If these clouds are true SSLs however, their source is not completely understood. One widely accepted scenario links SSLs with simultaneous existing sporadic E-layers in the ionosphere, whose high electron density recombines ionized $\mathrm{Na}^{+}$from ablated meteors and therefore creates in conjunction with wind shear on relatively short time scales a high Sodium density cloud (Collins et al. (2002)).

In addition these clouds show turbulent undulations, which swirl the Sodium around up to an extend of up to $5 \mathrm{~km}$ in height and creates challenges for the AO system to keep the LGS in focus. More information of sodium layer dynamics and MLT turbulence can be found in Pfrommer et al. (2009). 


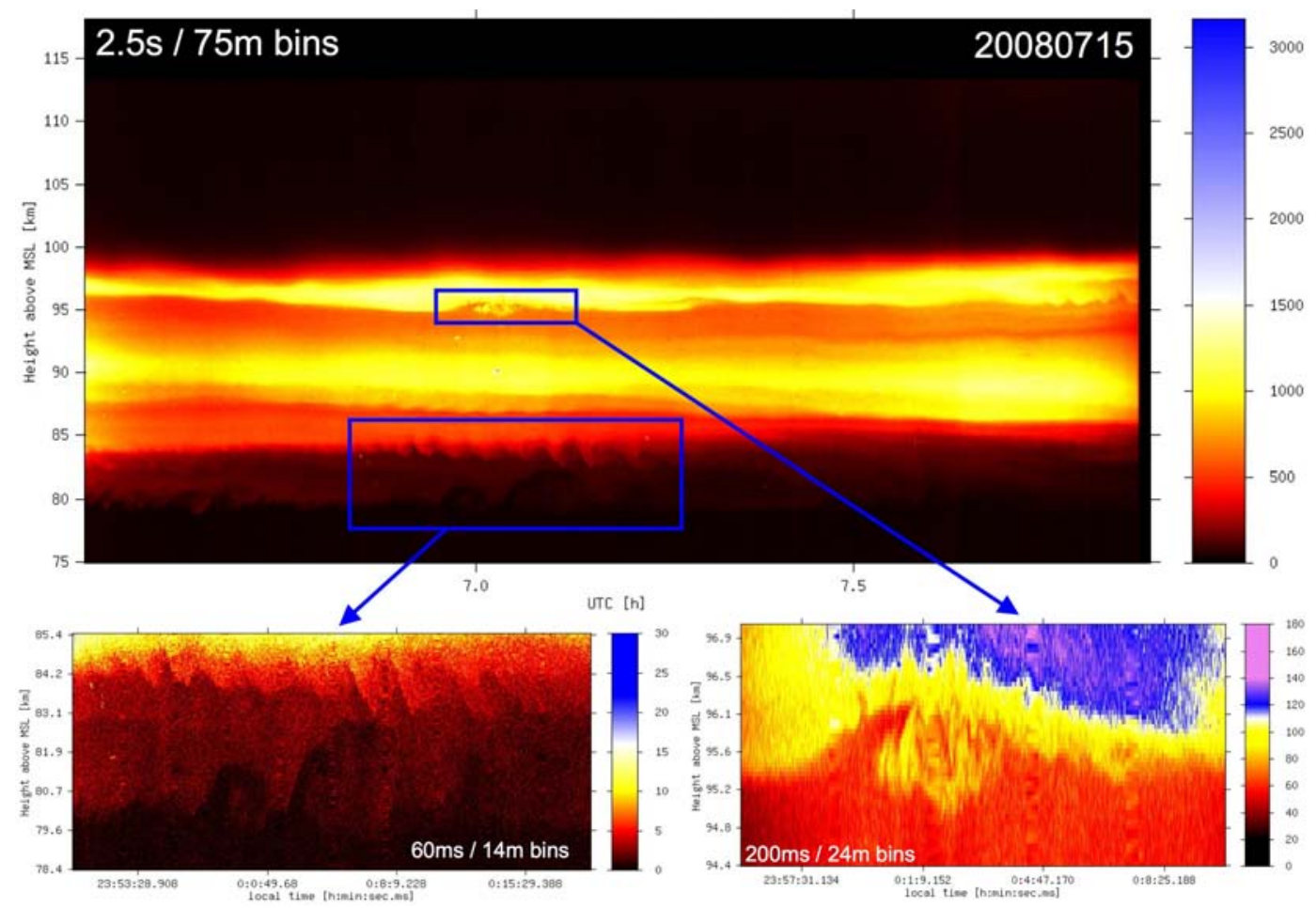

Fig. 1. Sodium layer density map with data from July $15^{\text {th }} 2008$. Color coded is the number of returned photons per bin $(2.5 \mathrm{~s} / 75 \mathrm{~m})$.

\subsection{Challenges with the Sodium Layer}

As indicated in the introduction, elongated sodium LGS are becoming a big problem for ELTs with their large primary mirror diameter $D$. For TMT, an LGS at the outer subaperture will have an elongation of almost 4". This challenges the centroid finding algorithms and also introduces systematic errors if the mean, intensity weighted, height of the sodium changes due to density fluctuations in the sodium layer profile. To overcome this problems, matched filtering algorithms are developed but their improvement depends on the degree of fine structure present within the sodium layer and its temporal variability as well as the impact of photon noise in the wavefront sensor (WFS), which scales with $D$ (for fixed laser power).

For this reason (and another: LGS cannot sense atmospheric "tip-tilt" fluctuations that change the position of the science target) it is essential that NGS also be employed. The available stars are generally very faint, sufficient only to provide the lowest order phase information (tiptilt and focus). This focus signal is noisy and has insufficient time resolution to allow the AO system to distinguish perfectly between focus errors caused by the atmosphere and those caused by variations in the sodium height. This focus error is also present for AO systems on current telescopes. It does however scale with $D^{2}$ and therefore only becomes severe for ELTs.

The rms wavefront error $\sigma_{r m s}$ due to a moving point source like a defocussing centroid motion at distance $h$ by $\Delta h$ is (Davis et al. (2006))

$$
\sigma_{r m s}=\frac{1}{16 \sqrt{3}}\left(\frac{D}{h}\right)^{2} \Delta h
$$

For TMT and a centroid located at $90 \mathrm{~km}$ height, moving by $46 \mathrm{~m}$, this wavefront error is $186 \mathrm{~nm}$, the entire TMT AO system's error budget. Of course this could be almost entirely reduced by the input of NGS, dependent on the timescales of the centroid motion. 
Therefore, variations in the sodium height, if sufficiently large on small timescales, can cause a significant loss of performance of the AO system and needed to be investigated to produce sodium profiles up to $25 \mathrm{~Hz}$.

\section{Results}

\subsection{The Mean Height Power Spectrum}

The main result, which is of great importance due to the above mentioned reasons, is the mean altitude power spectrum. Fig. 2 shows this for one night in red. The green power spectrum is the one derived from Davis et al. (2006). Above $1 \mathrm{~Hz}$, instrumental noise is obvious and we are currently working on this to reduce it to see the true dynamics up to the Nyquist limit of $25 \mathrm{~Hz}$.

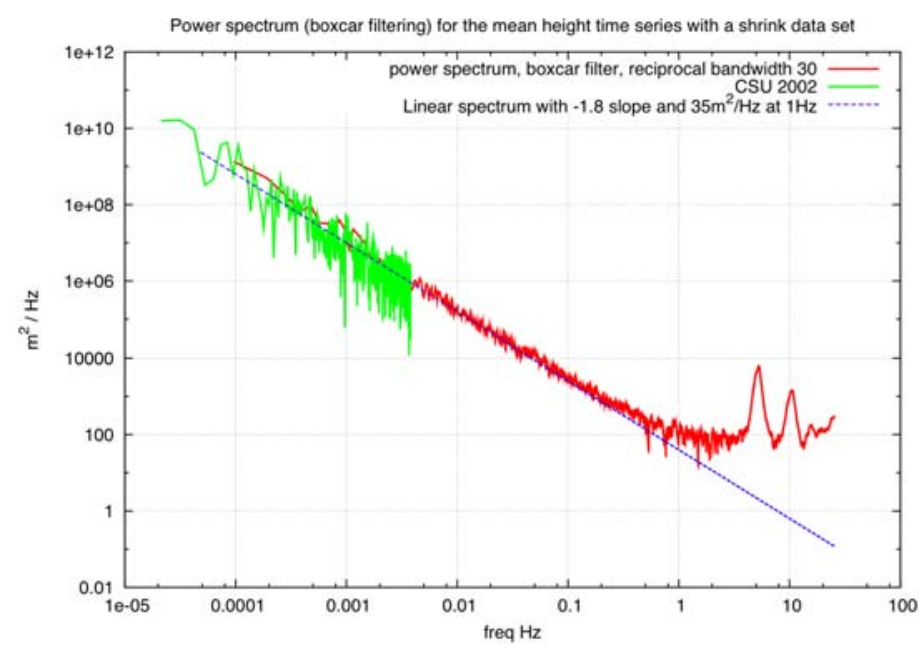

Fig. 2. Mean height power spectrum of one night, boxcar filtered.

The blue straight line follows a power law

$$
\Phi_{a}=35\left(\frac{v}{H z}\right)^{-1.8} \frac{m^{2}}{H z}
$$

From this equation the expected wavefront error due to focus error $\sigma_{w f e}$ can be derived via the variance of the mean height difference $\sigma_{h}^{2}$, also known as the time dependent structure function $D_{h}(t)$, which can be calculated using the autocorrelation function $B(t)$ and hence the power spectrum:

$$
\sigma_{h}^{2}=D_{h}(t)=2[B(0)-B(t)]=4 \int_{0}^{\infty} \Phi_{v} \sin ^{2}(\pi t v) d v=15.4298 \cdot \frac{A}{m^{2} / H z} \cdot t^{0.8} m^{2}
$$

Using eq. $1 \sigma_{w f e}$ can now be derived:

$$
\sigma_{w f e}=\frac{\sigma_{h}}{16 \sqrt{3}} \frac{D^{2}}{h^{2}}
$$

Having now two imaginary LGS separated by $30 \mathrm{~m}$ at $90 \mathrm{~km}$ height, what is the wavefront error? These two LGS obviously probe different spatial regions in the sodium layer and therefore have different centroid heights and hence focus error is introduced with only one focal stage. 
This quantity cannot be derived directly. We rather have to specify a period of time over which we want to get an estimate of the wavefront error. Under the Taylor frozen hypothesis and an assumed average wind speed throughout the sodium layer of $15 \mathrm{~m} / \mathrm{s}$ (Yuan et al. (2008)), we can get a first estimate of what LGS asterisms will statistically encounter. Using above numbers a tomographic error of $123 \mathrm{~nm}$ results. This is about $2 / 3$ of the entire TMT AO systems error budget.

It turns out that this error can be removed by the wavefront reconstruction algorithm that is developed by the TMT AO team (see papers from B.Ellerbroeck or L.Gilles in these proceedings) and a residual error of about $5 \mathrm{~nm}$ remains. This reduction applies due to the unique 2-deformable mirror design of TMT. Other AO designs have to be aware of this error and model this issue in detail with their design to address this potential trap.

Another area of potential problems could arise due to this error term. This is multi object adaptive optics (MOAO). As the AO corrected field of interest is hereby cut into several small subapertures, this tomographic error needs to be studied in detail and complete AO simulations with real time sodium profiles have to be used to find a solution.

\subsection{Meteor Trails}

Prior to the UBC lidar system, there have been reports of detection of meteor trails, showing short and locally confined photon return bursts as much as 10 times more than the surrounding average sodium layer return (Kane \& Gardner (1993), von Zahn et al. (1999)). These spikes in the data are caused by incoming larger meteors in the $\mu$ g-range, whose plume drifts over the lidar beam. Due to the coarse time resolution these studies only picked out events arising from larger meteors and a population in the minute range. With our lidar, we are able to detect and show that they can last as short as a few 100 s of ms. We are able to detect about 20 of the short lasting events per night.

Their occurrence tends to show up in the lower half of the sodium layer below $87 \mathrm{~km}$ and we are currently working to asses the impact of the whole population to AO systems. The main question hereby is to quantify their ability to change the centroid height of the instantaneous sodium profile and the impact on AO systems, dependent on their size (in height and temporal extension), their centroid distance, seasonal occurrences and number counts and their diffusion status.
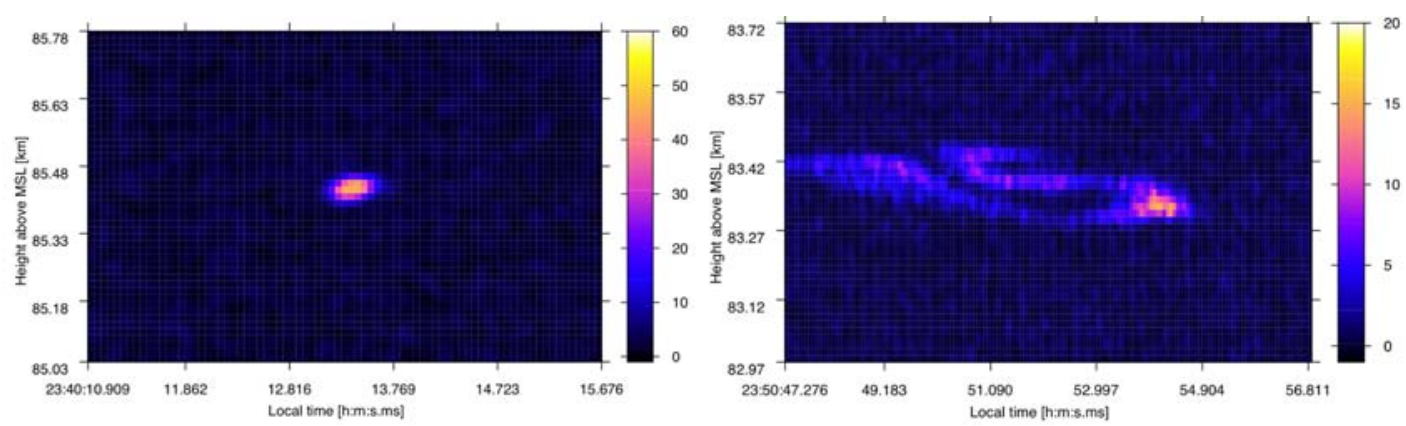

Fig. 3. Meteor trails drifting across the laser beam on $15^{\text {th }}$ July 2008.

Fig. 3 shows two such events. The event on the left side is still round and therefore young, hence not yet dissipated, whereas on the right side, an event from the same night, just 10 minutes later shows severe diffusion and the effect of the mesospheric wind and wind shear.

In addition to these short lasting events (on a minute, second and subsecond time scale) there is evidence that the already discussed (section 2.1) sporadic sodium layers (SSL) show relatively short fluctuations in density on a minute time scale (Liu \& Yi (2009)). This is also under current investigation with our data set and the impact on AO systems will be addressed. 


\section{Conclusion}

The UBC lidar system has been build to investigate the sodium layer at time scales up to $25 \mathrm{~Hz}$, relevant for future AO systems and asses the impact of sodium density variations.

The main aspects of the systems are:

- High spatio-temporal resolution profiles used in development and improvement of AO-systems $\Rightarrow$ Software development, dynamic focussing

- Temporal changes in the sodium density structure $\Rightarrow$ Variations of the substructure of elongated LGS $\Rightarrow$ centroid errors

- Mean sodium altitude variation $\rightarrow$ wavefront errors in focus and higher-order symmetric modes

- Tomographic errors due to different (unknown) mean altitudes for different LGS in the asterism $\Rightarrow$ No problem for the TMT AO system, but potential impact on MOAO?

- Errors in the uplink beam pointing will couple into sodium altitude errors due to the spatial variability of the mean altitude

- Impact of short time scale density enhancements due to meteor trail drifts across the laser beam as well as variations in SSLs is an area of current investigation

In order to asses the impact of the tomographic error in more detail we are currently upgrading the system by adding a tip-tilt stage to the laser uplink. This will enable us to simulate LGS asterisms and also get direct wind and wind shear information. The resulting horizontal sodium map will be the first of its kind and should reveal more details of this interesting region.

The UBC lidar system has proven to deliver useful information for the design of next generation AO systems as well as to improve the current understanding in the MLT region that shows highly dynamic weather behavior, also important for the global atmospheric energy exchange.

\section{References}

Ceplecha, Z., Borovička, J., Elford, W. G., Revelle, D. O., Hawkes, R. L., Porubčan, V., Šimek, M.: 1998, Space Science Reviews 84, 3271

Clemesha, B. R.: 1995, Journal of Atmospheric and Terrestrial Physics 57, 7252

Collins, S. C., Plane, J. M. C., Kelley, M. C., Wright, T. G., Soldán, P., Kane, T. J., Gerrard, A. J., Grime, B. W., Rollason, R. J., Friedman, J. S., González, S. A., Zhou, Q., Sulzer, M. P., Tepley, C. A.: 2002, Journal of Atmospheric and Solar-Terrestrial Physics 64, 8452

Davis, D. S., Hickson, P., Herriot, G., She, C.-Y.: 2006,Optics Letters 31, 3369 1, 3, 4

Hecht, J. H., Kovalam, S., May, P. T., Mills, G., Vincent, R. A., Walterscheid, R. L., Woithe, J.: 2004, Journal of Geophysical Research (Atmospheres) 109, 202

Herriot, G., Hickson, P., Ellerbroek, B., Véran, J.-P., She, C.-Y., Clare, R., Looze, D.: 2006, vol. 6272 of Society of Photo-Optical Instrumentation Engineers (SPIE) Conference Series 1

Hickson, P., Pfrommer, T., Cabanac, R., Crotts, A., Johnson, B., de Lapparent, V., Lanzetta, K. M., Gromoll, S., Mulrooney, M. K., Sivanandam, S., Truax, B.: 2007, PASP 119, 4442

Kane, T. J., Gardner, C. S.: 1993, Science 259, 12975

Liu, Y., Yi, F.: 2009, Journal of Atmospheric and Solar-Terrestrial Physics 71, 13745

Pfrommer, T., Hickson, P., She, C.-Y.: 2009, Geophys. Res. Lett. 36, 158312

Pfrommer, T., Hickson, P., She, C.-Y., Vance, J. D.: 2008, vol. 7015 of Society of Photo-Optical Instrumentation Engineers (SPIE) Conference Series 2

Plane, J. M.: 2003, AGU Fall Meeting Abstracts , A2+ 2

von Zahn, U., Gerding, M., Höffner, J., McNeil, W. J., Murad, E.: 1999, Meteoritics and Planetary Science 34, 10175

Xu, J., Smith, A. K., Collins, R. L., She, C.-Y.: 2006, Journal of Geophysical Research (Atmospheres) 111, 173012

Yuan, T., Schmidt, H., She, C. Y., Krueger, D. A., Reising, S.: 2008, Journal of Geophysical Research (Atmospheres) 113, 20103 2, 5 